\title{
Fluidization of Transient Filament Networks
}

\author{
Fanlong Meng $g^{\dagger, \ddagger}$ and Eugene M. Terentjev ${ }^{*, \ddagger}$ \\ $\dagger$ Rudolf Peierls Centre for Theoretical Physics, University of Oxford, Oxford OX1 3NP, \\ U.K. \\ $\ddagger$ Cavendish Laboratory, University of Cambridge, JJ Thomson Avenue, Cambridge CB3 \\ OHE, U.K. \\ E-mail: emt1000@cam.ac.uk
}

\begin{abstract}
Stiff or semiflexible filaments can be crosslinked to form a network structure with unusual mechanical properties, if the crosslinks at network junctions have the ability to dynamically break and re-form. The characteristic rheology, arising from the competition of plasticity from the transient crosslinks and nonlinear elasticity from the filament network, has been widely tested in experiments. Though the responses of a transient filament network under small deformations are relatively well understood by analyzing its linear viscoelasticity, a continuum theory adaptable for finite or large deformations is still absent. Here we develop a model for transient filament networks under arbitrary deformations, which is based on the crosslink dynamics and the macroscopic system tracking the continuously re-shaping reference state. We apply the theory to explain the stress relaxation, the shape recovery after instant deformation, and the necking instability under a ramp deformation. We also examine the role of polydispersity in the mesh size of the network, which leads to a stretched exponential stress relaxation and a diffuse elastic-plastic transition under a ramp deformation. Although dynamic crosslinks are taken as the source of the transient network response, the model can be easily adjusted to incorporating other factors inducing fluidization, such as filament
\end{abstract}


breakage and active motion of motor crosslinks, opening a way to address cell and tissue activity at the microscopic level.

\section{Introduction}

Transient filament networks are ubiquitous in biology, such as cytoskeleton, extracellular matrix (ECM), and macroscopically in connective tissue. ${ }^{1]}$ Quantitative understanding of their mechanical responses is very important from both the scientific and the biotechnology viewpoints. $\frac{213}{}$ Such networks could be made of various kinds of stiff and semiflexible filaments (F-actin, collagen, vimentin, neurofilament, etc.), and the transient properties of the networks originated from the dynamic nature of the crosslinks, such as myosin, filamin, actinin, etc., where the crosslinks can be dynamically broken or adjusted, spontaneously or under stress. Existence of such networks is essential in achieving specific biological functions, such as adaptation and migration of cells, and contraction and recovery of tissues. Abnormalities, such as tissue injury (in fascia and tendon) or tumor growth, are usually due to the onset or the prevention of the network fluidization (stress-induced plastic flow) of a specific type of transient filament networks.

Experimentally, the rheology of a transient filament network is investigated widely, $\underline{4}[6$ with particular attention to stress relaxation arising from crosslink breakage under constant deformation, ${ }^{7-9}$ and stress softening and yield upon ramp deformation with constant strain rate. $10+12$ There are several revealing phenomena found in such networks. Murakami et al. 13 studied the reshaping of red blood cells due to adaption of the cytoskeleton by constraining cells in a narrow tube. Wang et al. ${ }^{14}$ observed necking when uniaxially stretching the cardiac tissue within the collagen/fibrin matrix.

Theoretically, modelling of transient polymer networks can date back to $1940 \mathrm{~s}, \frac{15}{15}$ when breakage and re-formation of crosslinks are introduced into a polymer network. This concept has now been broadly adopted in modelling transient flexible polymer networks. Specifical- 


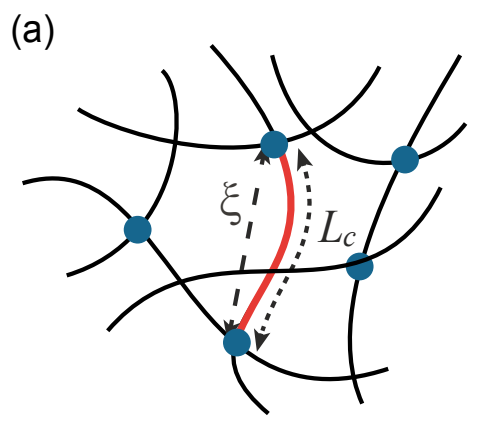

: breakable crosslinks (b)
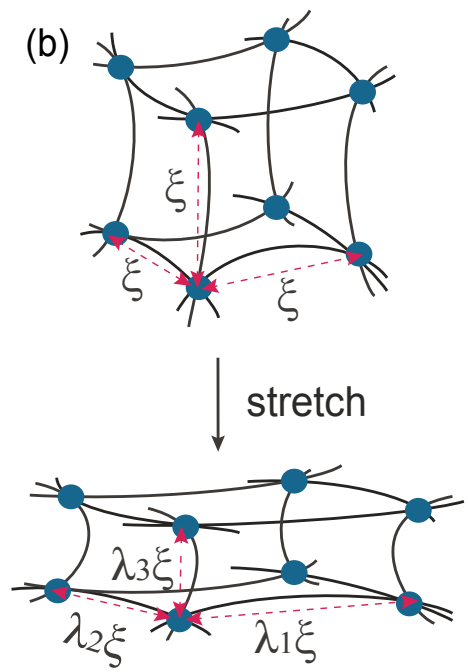

Figure 1: (a) Sketch of a filament network, with the end-to-end length $\xi$ treated as the mesh size, and $L_{c}$ the contour length of the filament connecting two neighboring crosslinks. (b) 3 -chain model of filament network before and after deformation, with average mesh size $\xi$ undergoing a locally affine deformation.

ly, Tanaka and Edwards $\stackrel{16}{16}$ formulated a statistical theory for the transient flexible polymer network from the microscopic viewpoint, where the energy of the material is obtained by summing up those from flexible polymers with different reference states due to the crosslink dynamics. Drozdov ${ }^{17}$ and Long et al. $\frac{18 \mid 19}{19}$ used different approaches, in effect replacing the statistical analysis of Tanaka-Edwards with known elastic models of permanent networks, but obtained essentially the same final results on the network dynamics. The Tanaka-Edwards ideas were further developed by Meng and Terentjev, 20 and a continuum energy form of transient flexible polymer networks was obtained by integrating the classical rubber-elastic free energy of permanent flexible polymer networks with the distribution of dynamic reference states and probabilities. With the help of that model, the rheology of transient flexible polymer networks in the regime of large deformation was also studied, where the limited extensibility of polymer strands was accounted for ${ }^{21}$. Although many aspects of transient networks are shared between the flexible network at large deformations, and the network of rigid/semiflexible filaments, which also have a characteristic divergence when extension ap- 
proaches the limit, the semiflexible nature of the crosslinked filaments adds several important distinctions. The elasticity of a permanently crosslinked filament network is well-studied, where the free energy of the network is obtained by summing up the contributions from filaments with different network topologies. $\frac{213}{2}$ Due to the interplay between the bending rigidity and the entropic contribution, filament networks exhibit some characteristic features, such as the 3/2-scaling of their stress-stiffening, the limited mechanic stability, negative normal stress, etc. Compared with the mature understanding of transient flexible networks and permanent filament networks, the rheology of a transient filament network has not yet been theoretically described, mainly due to the complexity in the coupling between the nonlinear elasticity from the filaments and the plasticity from the crosslink dynamics.

From the theoretical viewpoint, linear viscoelasticity is the most studied issue in which the nonlinear elasticity of the network upon large deformations is absent. As an essential outcome of linear viscoelasticity, the dependence of the storage (loss) modulus of the material on the deformation frequency is discussed in different ways, for example, traditional rheology analyses incorporating crosslink breakage and filament fluctuations by Lieleg et al., ${ }^{5}$ the Langevin-dynamics model incorporating breakage of transient crosslinks by Broedersz et al., $\underline{6}$ the generalized Maxwell model assuming polydispersity in stress relaxation rate by Unterberger et al., 22 and the glassy worm-like chain model by Kroy et al. $\stackrel{23 \mid 24}{2}$ These models, though different in their approaches, manage to capture how a transient filament network responses under small deformations, by incorporating crosslink dynamics in the linear regime of the dynamic mechanical response of the system.

For large deformations, a good atempt was made by López-Menéndez and Rodríguez 25 in handling transient filament networks under finite shear deformations, where the authors adapted the 8-chain model of permanent filament network by Palmer and Boyce ${ }^{26}$ (all filaments are treated identical in the model), by making contour lengths of subfilaments bridging neighboring crosslinks change with time due to breakage of crosslinks. The theory nicely captures how this systems responds under a simple shear, by incorporating the interplay 
between network elasticity and plasticity due to the dynamic crosslinks, although the mer-

its of the 8-chain model to describe a filament network have been questioned. $\stackrel{20}{ }$ Although such theoretical attempts help in understanding certain aspects of the dynamic mechanical responses of transient filament networks, such as linear viscoelasticity in small deformation regime and rheology under simple shear deformation, a simple but general continuum theory for transient filament network under arbitrary deformations is still lacking.

Based on the continuum models of transient flexible networks ${ }^{20}$ and of permanent filament networks, 27 we provide a continuum energy form of a transient filament network. Since the underlying model of the individual filament that we use has no approximations, the resulting theory is applicable for the rheology of a transient filament networks not only for small, but also for finite or large deformations. As applications of the general theory, we first discuss how a transient filament network with monodispersed mesh size responds in a uniaxial tensile experiment, considering the stress relaxation under a constant strain and its shape recovery after releasing, and the elastic-plastic transition under a ramp deformation at constant rate. We then show the role of the distribution (polydispersity) of mesh sizes, in producing a realistic dynamical response closely matching what is observed in experiments.

\section{Model}

\section{Permanent filament network}

Mechanical properties of a single filament have been extensively studied, since Kratky and Porod proposed the worm-like chain model in 1949.28 Consider the sub-filament (colored red) bridging two neighboring crosslinks in Fig. 1(a). If its configuration is parameterized by the position $\boldsymbol{r}(s)$, where $0 \leq s \leq L_{c}$ denotes the arc-length coordinate of the filament ( $L_{c}$ is the contour length between crosslinks), then the end-to-end distance is $\xi=\mid \boldsymbol{r}\left(L_{c}\right)-$ $\boldsymbol{r}(0) \mid$. In the simplest model, the bending energy of a filament is determined by the local curvature $\frac{1}{2} \kappa \int_{0}^{L_{c}} d s\left|\boldsymbol{r}^{\prime \prime}(s)\right|^{2}$, where $\kappa$ is the bending modulus, which defines the persistence 
length, $l_{p}=\kappa / k_{\mathrm{B}} T$. When $l_{p} \sim L_{c}$, the semiflexible filament has the most unusual physical properties; curiously, this is frequently the case of biological filaments. ${ }^{27}$ In spite of many good attempts, there is no analytical expression for the free energy of a rigorously inextensible filament. However, a closed analytical form is now available if one accepts a limit of 'mean inextensibility', that is, when the chain maintains its constant contour length $L_{c}$, while locally each segment is inextensible on average. Although mathematically this is an approximation - physically this limit is perhaps a more reasonable model of a semiflexible chain. Within this framework, Blundell et al. ${ }^{[29}$ obtained an expression for the free energy of semiflexible filament, which has no other approximations, i.e. remaining valid across the full range from the rigid-rod to the flexible-chain regimes. This free energy can be written as a function of the end-to-end factor, $x=\xi / L_{c}$ (see Fig. 1):

$$
F_{\mathrm{sf}}(x ; c)=k_{\mathrm{B}} T \pi^{2} c\left(1-x^{2}\right)+\frac{k_{\mathrm{B}} T}{\pi c\left(1-x^{2}\right)},
$$

where $c=l_{p} / 2 L_{c}$ is a non-dimensional measure of relative stiffness of the filament: no matter how locally stiff the filament might be - it will behave as a flexible chain if its contour length $L_{c}$ is long enough. The tension force is acting on this filament along its end-to-end vector $\xi: f=\partial F_{\mathrm{sf}} / \partial \xi$, if this distance deviates from the 'natural length' in the force-free state. This natural length of a semiflexible filament is $x_{0}=\sqrt{1-1 / \pi^{3 / 2} c}$, which means that locally flexible filaments with $c<\pi^{-3 / 2}$ (i.e. $l_{p}<0.36 L_{c}$ ) have no natural length: as in a classical polymer chain, any end-to-end extension leads to a tensile force. For given $c$, if $x$ is larger than the equilibrium value $x_{0}(c)$, then the filaments in the network are pre-stretched; otherwise, the filament is pre-compressed; see ${ }^{2729}$ for detail.

The constitutive model that produces a macroscopic continuum elastic free energy of a random network, and the corresponding equilibrium stress-strain relationship, has been discussed for many years. $\frac{30131}{1 n}$ our recent study of an equilibrium network of semiflexible filaments, ${ }^{27}$ we choose a 3 -chain constitutive model, whose validity is tested by quantitatively 
fitting experimental stress-strain relationship for a broad range of filament networks, and by

illustrating negative normal stress as a characteristic property for filament network,, 32133 and by the analysis of marginal rigidity ${ }^{34}$ The idea of the 3 -chain model is very simple. ${ }^{30}$ Suppose that the network is distorted with a deformation tensor $\boldsymbol{E}$, and the elongation ratios along three principal stretching directions $\boldsymbol{e}_{1,2,3}$ (the eigenvectors of $\boldsymbol{E}$ ) are $\lambda_{1,2,3}$, respectively. A 3-chain cubic cell can be constructed as shown in Fig. 1(b), where its nodes represent the crosslinks, and its edges represent the end-to-end segments of the filaments connecting neighboring crosslinks. The cell is aligned along the principle direction of the deformation tensor. On deformation, the lengths of the three orthogonal edges will change from $\xi$ (the average mesh size) to $\lambda_{i} \xi$, respectively. The macroscopic network can then be built by repeating such a 3 -chain cubic over the whole space, assuming the overall affine deformation and reflecting the symmetry of its principal extensions. Other constitutive models, such as 1-chain and 8-chain model, have been shown to produce inadequate results in a filament network. ${ }^{27}$

Suppose the network consists of filaments with stiffness $c$ and end-to-end factor $x=\xi / L_{c}$, which in effect gives information about filament pre-tension between crosslinks (when $x$ deviates from $x_{0}$ ). The macroscopic free energy density of such a network, in the 3-chain model, is given by multiplying the crosslink density, $n / 3$ (where $n$ is the number density of the sub-filaments), with the sum of the energy contributions from three orthogonal chains. After some algebra, this energy density can be expressed as a function of the three invariants of deformation tensor, producing a compact result,

$$
F_{\text {p.n }}=\frac{n k_{\mathrm{B}} T}{3}\left[\pi^{2} c\left(3-x^{2} I_{1}\right)+\frac{3-2 I_{1} x^{2}+I_{2} x^{4}}{\pi c\left(1-I_{1} x^{2}+I_{2} x^{4}-I_{3} x^{6}\right)}\right],
$$

where $I_{1}=\lambda_{1}^{2}+\lambda_{2}^{2}+\lambda_{3}^{2}, I_{2}=\lambda_{1}^{2} \lambda_{2}^{2}+\lambda_{2}^{2} \lambda_{3}^{2}+\lambda_{3}^{2} \lambda_{1}^{2}$, and $I_{3}=\lambda_{1} \lambda_{2} \lambda_{3} \cdot{ }^{355}$ For macroscopically incompressible materials, there is a constraint $I_{3}=1$, which will be used in the following discussion. $F_{\mathrm{p} . \mathrm{n}}$ is Helmholtz free energy of the material, and Gibbs free energy can be 
introduced instead as $G_{\mathrm{p} . \mathrm{n}}=F_{\mathrm{p} . \mathrm{n}}+p \sqrt{I_{3}}$, by introducing a Lagrangian multiplier $p$, playing the role of local pressure in charge of the incompressibility. However, we will only consider the practical situations where the boundary conditions or the local pressure are not of interest, and therefore stay with the Helmholtz formulation with a rigid constraint $I_{3}=1$.

The chains in a crosslinked network can be (on average) in either pre-stretched, precompressed, or in force-free state. The mechanical stability of the network is determined by the value of its linear elastic modulus, defined as $\mu_{i j}=\partial^{2} F / \partial E_{i j}^{2}$ at zero deformation, and we have earlier shown that the boundary of marginal rigidity (when $\mu=0$ for isotropic material) lies very close to the force-free condition $x_{0}(c) . \underline{27}$

\section{Transient filament network}

In a typical transient network, filaments can be broken from their crosslinks and then reconnected in new positions in some cases. For the breakage of a biologically relevant crosslinker, there are two main models. One model ${ }^{[36}$ assumes that the breakage rate of a crosslink without any external force, $\beta_{0}$, is defined by an activation law $\beta_{0}=\omega_{0} e^{-G_{b} / k_{\mathrm{B}} T}$, where $\omega_{0}$ is a thermal oscillation frequency of filaments, and $G_{b}$ is the energy barrier for a filament to break from a crosslink. When there is a force acting on the chain, the breakage rate will increase: $\beta=\beta_{0} e^{f a / k_{\mathrm{B}} T}$, with the length $a$ representing the crosslink size, or equivalently, the filament diameter. This is effectively the Bell law ${ }^{\sqrt{36}}$ reproducing the classical Smoluchowski analysis. As an example, for a heavy meromyosin (HMM) in the rigor state bonded with actin (actomyosin bond in its rigor state), $\frac{37}{3} \beta_{0}$ is of the order of $1 s^{-1}$ and the length $a$ is of the order of $1 \mathrm{~nm}$. For simplicity, the absolute value of the force will be used, regardless of compressing or stretching. The other model assumes the breakage rate is a dual exponential function caused by so-called "catch and slip" bond: $\frac{37 \mid 38}{38} \beta=\beta_{0}^{c} e^{-f d_{c} / k_{\mathrm{B}} T}+\beta_{0}^{s} e^{f d_{s} / k_{\mathrm{B}} T}$, where $\beta_{0}^{c}$ and $d_{c}$ are the breakage rate and characteristic length of a "catch" bond, $\beta_{0}^{s}$ and $d_{s}$ are the breakage rate and characteristic length of a "slip" bond. For an actomyosin bond in its rigor state, it is reported by Guo et al. that, $\stackrel{37}{3}$ for the catch bond, $\beta_{0}^{c}$ is of the order of $100 s^{-1}$ and 
the length $d_{c}$ is about $1 \mathrm{~nm}$, and for the slip bond, $\beta_{0}^{s}$ is of the order of $10 \mathrm{~s}^{-1}$ and the length $d_{s}$ is of the order of $0.1-1 \mathrm{~nm}$. In this way, the breakage rate of a crosslink depends on a competition between the two bonds. Usually, $d_{c}$ is much larger than $d_{s}, \stackrel{37138}{\text { meaning that }}$ the "catch" bond dominates in the case of small force in a relatively narrow range, while the "slip" bond can determine how a crosslink responds under large forces in a relatively wide range; when the deformation of the network is large, the force acting on the filaments is also large, and in this case, the simple "slip" bond is an even better approximation. Furthermore, for seeking the transparency of our model in the following discussion, we will stay with the first simple model possessing a single exponential function (purely the "slip" bond model) for the dynamics of crosslinks in a transient filament network.

The rate of re-crosslinking, $\frac{39}{39}$ less controversially, is defined as $\rho_{0}=\omega_{0} e^{-G_{c} / k_{\mathrm{B}} T}$, where thermal oscillation frequency $\omega_{0}$ is same as that in $\beta_{0}$, and $G_{c}$ is the lower energy barrier for a dangling filament segment to be re-attached to a crosslink. It may be possible that the re-crosslinking rate can be also dependent on the force acting on the chain, due to changed orientations of chains under deformation, however, this effect is ignored here for simplicity. In contrast, the diffusion of a dangling chain end in order to "find" a free crosslinking site might be very influential. Suppose a characteristic diffusion time for this process is $\tau$, then the effective re-crosslinking rate is $\rho=\rho_{0} /\left(1+\rho_{0} \tau\right)$, showing that the reaction rate $\rho_{0}$ dominates when the diffusion time is short, while $\rho \simeq 1 / \tau$ when the diffusion time is long.

Suppose that total number of chains in the network is $N_{t o t}$, with $N_{c}(0)$ chains crosslinked at time $t=0$, and $N_{b}(0)=N_{t o t}-N_{c}(0)$ chains are uncrosslinked (dangling). As a function of the force acting on the chain, the breakage rate of the crosslinks in a transient network can only be defined locally and dynamically in a configuration that depends on both the current state at time $t$ and the reference/crosslinking state at time $t^{\prime}$. At time $t$, the breakage rate of the crosslinks formed at time $t^{\prime}$, will be denoted as $\beta_{t ; t^{\prime}}$. After an infinitesimal interval $\Delta t$ following $t=0$, the number of the chains remaining crosslinked from the initially crosslinked network will decrease to $N_{c}(0)\left[1-\beta_{0 ; 0} \Delta t\right] \simeq N_{c}(0) \exp \left[-\beta_{0 ; 0} \Delta t\right]$ due to the breakage of the 
crosslinks at time $t=0$. At the same time, there will be $N_{b}(0) \rho \Delta t$ chains re-connected into the network. The total number of the chains crosslinked at time $\Delta t$ is the sum of these two contributions: $N_{c}(0) \exp \left[-\beta_{0 ; 0} \Delta t\right]+N_{b}(0) \rho \Delta t$. After another time interval, at $t=2 \Delta t$, the number of the crosslinked chains surviving from the beginning decreases further to $N_{c}(0) \exp \left[-\beta_{0 ; 0} \Delta t-\beta_{\Delta t ; 0} \Delta t\right]$. The number of chains which were crosslinked during the first time interval decreases due to new breaking to $N_{b}(0) \rho \Delta t \exp \left[-\beta_{\Delta t ; \Delta t} \Delta t\right]$. The number of newly crosslinked chain during the second time interval is $N_{b}(\Delta t) \rho \Delta t$. The total number of the crosslinked chains at time $t=2 \Delta t$ is, therefore:

$$
N_{c}(0) \exp \left[-\beta_{0 ; 0} \Delta t-\beta_{\Delta t ; 0} \Delta t\right]+N_{b}(0) \rho \Delta t \exp \left[-\beta_{\Delta t ; \Delta t} \Delta t\right]+N_{b}(\Delta t) \rho \Delta t
$$

By repeating this process, the total number of the crosslinked chains after $N$ time intervals will become

$N_{c}(N \Delta t)=N_{c}(0) \exp \left[-\sum_{i=0}^{N-1} \beta_{t=i \Delta t ; 0} \Delta t\right]+\sum_{j=0}^{N-1} N_{\mathrm{b}}(j \Delta t) \rho \Delta t \exp \left[-\sum_{k=j+2}^{N} \beta(k \Delta t ;[j+1] \Delta t) \Delta t\right]$,

with its continuous form being:

$$
N_{c}(t)=N_{c}(0) \exp \left[-\int_{0}^{t} \beta\left(t^{\prime} ; 0\right) d t^{\prime}\right]+\int_{0}^{t} N_{\mathrm{b}}\left(t^{\prime}\right) \exp \left[-\int_{t^{\prime}}^{t} \beta\left(t^{\prime \prime} ; t^{\prime}\right) d t^{\prime \prime}\right] \rho d t^{\prime}
$$

The first term in the right-hand side denotes the number of the crosslinked chains surviving from the beginning, while the second term counts the chains re-crosslinked between $t=0$ and $t$ that are still surviving at the present time. As the reference states of the individual chains are defined according to when they are crosslinked, the elastic free energy density of the transient network is given by a retarded (memory) expression:

$$
F_{\mathrm{t} . \mathrm{n}}(t)=\exp \left[-\int_{0}^{t} \beta\left(t^{\prime} ; 0\right) d t^{\prime}\right] F_{\mathrm{p} . \mathrm{n}}(t ; 0)+\int_{0}^{t} \frac{N_{\mathrm{b}}\left(t^{\prime}\right)}{N_{c}(0)} \exp \left[-\int_{t^{\prime}}^{t} \beta\left(t^{\prime \prime} ; t^{\prime}\right) d t^{\prime \prime}\right] F_{\mathrm{p} . \mathrm{n}}\left(t ; t^{\prime}\right) \rho\left(d t^{\prime}\right)
$$


This is a generic framework developed in, ${ }^{20}$ which will now be adapted to the network of semiflexible filaments by choosing the Eq. (2) for the $F_{\text {p.n }}\left(t ; t^{\prime}\right)$. Note that similarly with the Rouse-type relaxation in rubbers of crosslinked flexible chains, $\frac{40}{4}$ a permanent filament

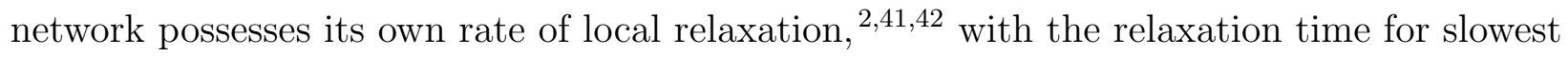
mode as $\eta L_{c}^{4} /\left[\kappa 4 \pi^{3} \ln \left(A L_{c} / a\right)\right]$, where $A$ is a constant of order unity and $\eta$ denotes the viscosity. For a filament network with typical mesh size $\sim 10 \mu m$, the relaxation time of the slowest mode is about $1 \mathrm{~s}$, which is much smaller than the lifetime of a crosslink in a filament network, $\left(\sim 10 s\right.$ in experiment on red blood cell ${ }^{[13}$ and $\sim 10^{3} s$ of bundled cytoskeleton networks $\left.{ }^{8}\right)$. As a result, we conclude that the mechanic relaxation process of individual filaments is too fast for our range of interest, and will not be considered in this work.

The elastic energy in Eq. (4) is determined by the deformation tensor $\boldsymbol{E}\left(t ; t^{\prime}\right)$ measured with respect to the reference state established at $t=t^{\prime}$, which is expressed by $\boldsymbol{E}\left(t ; t^{\prime}\right)=$ $\boldsymbol{E}(t ; 0) \cdot \boldsymbol{E}^{-\mathbf{1}}\left(t^{\prime} ; 0\right)$. This time-dependent strain also determines the local force that affects the rate of crosslink breaking in the exponents of activation rate: $\exp \left[-\int_{t^{\prime}}^{t} \beta\left(t^{\prime \prime} ; t^{\prime}\right) d t^{\prime \prime}\right]$. By assuming that the material is incompressible, we can obtain the constitutive stress-strain relation of the transient filament network, as the derivative $\boldsymbol{\sigma}_{i j}^{\text {ela }}=\partial F_{\mathrm{t} . \mathrm{n}} / \partial E_{i j}$, since there is no difference between the Gibbs free energy $G_{\mathrm{t} . \mathrm{n}}=F_{\mathrm{t} . \mathrm{n}}(t)+p \operatorname{det}[\boldsymbol{E}]$, and the Helmholtz $F_{\mathrm{t} . \mathrm{n}}$, when we use the rigid constraint $I_{3}=\operatorname{det}[\boldsymbol{E}]=1$. Note that there are also various

microscopic models $\frac{16143}{10}$ for the transient network, especially transient rubber, which deal with the mechanical properties from the statistical viewpoint.

\section{Results and Discussions}

By taking a uniaxially stretched material as our example system, we start with the analysis of stress relaxation of a transient network of monodisperse filaments under an instant strain. We then examine how a transient filament network responds to a ramp extension with a constant strain rate, illustrating three regimes: elastic, plastic flow, and a necking instability 
region separating the two. After this, we extend the discussion to a polydisperse transient filament network, where a non-exponential stress relaxation and a different stress-strain relation under dynamic extension are found.

\section{Monodisperse network}

We start with a simplifying assumption of monodisperse filament network, where the mesh size is assumed uniform across the whole network. Assuming that the stiffness of filaments is also the same, we can then have a network with a uniform pre-tension, characterized by a factor $x$ as in Eq. (2). The deformation tensor in an incompressible uniaxial extension experiment is: $\boldsymbol{E}=\lambda \boldsymbol{e}_{x} \boldsymbol{e}_{x}+1 / \sqrt{\lambda}\left(\boldsymbol{e}_{y} \boldsymbol{e}_{y}+\boldsymbol{e}_{z} \boldsymbol{e}_{z}\right)$, where $\lambda$ is the elongation ratio along the stretch direction, $\boldsymbol{e}_{x}$. The elongation ratios of the other two orthogonal directions, $\boldsymbol{e}_{y}$ and $\boldsymbol{e}_{z}$, are equal to $1 / \sqrt{\lambda}$, due to the assumed incompressibility. In this case, the invariants of the deformation tensor are: $I_{1}=\lambda^{2}+2 / \lambda, I_{2}=2 \lambda+1 / \lambda^{2}$ and $I_{3}=1$. Then the filament free energy in Eq.(2), can be expressed as a function of stretch ratio, $\lambda$. Because the stretch ratios along the three orthogonal directions are different, forces acting on chains in the cubic cell are also different, even if the chains were all crosslinked at the same time $(t=0)$. When one chain breaks from a crosslink, then the other two chains sharing the same crosslink are able to move freely along the direction of the chain detached from this crosslink. Therefore, they can achieve a force-free equilibrium state with a much lower breakage rate; the crosslink becomes elastically 'inactive' and does not contribute any elastic energy to the total network. Thus for simplicity, we calculate the breakage rate of the crosslink with the largest force acting on the three orthogonal chains, assuming it breaks first and the other chains become elastically inactive afterwards.

\section{Stress relaxation and shape recovery}

Murakami et al. ${ }^{[13}$ conducted a shape-recovery experiment on red blood cells. First, a cell is squeezed in its radial direction into a narrow slit for a period of $t$ (order of minutes). Then, 
(a)

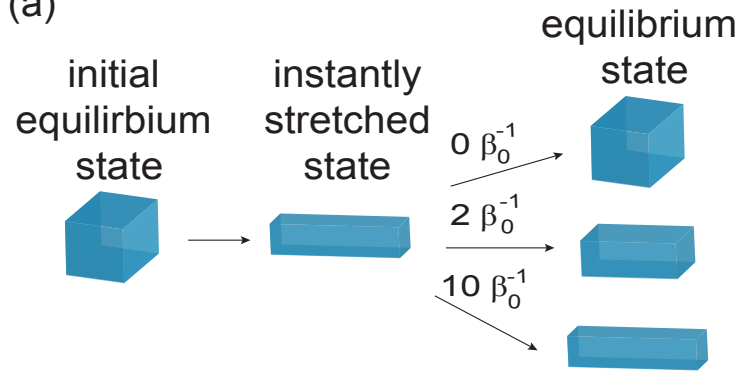

(b) 1.0

(b)

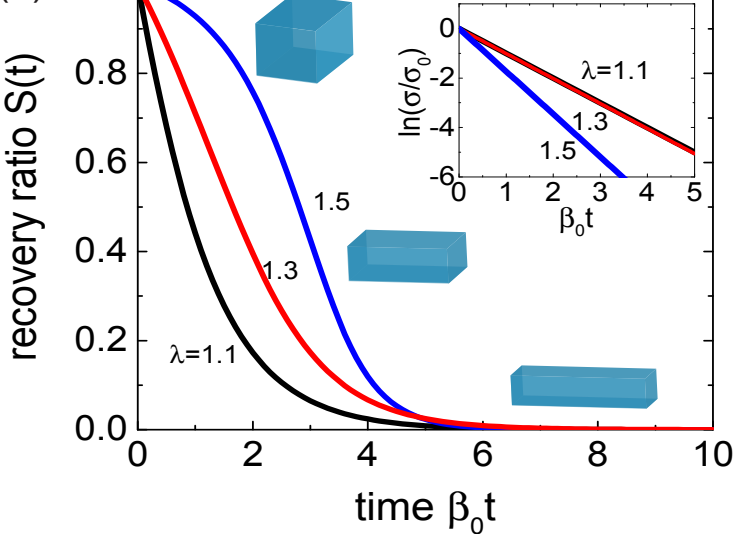

Figure 2: (a) A cube of transient filament network stretched at $t=0$, kept being stretched for a period of $0,2 / \beta_{0}$ and $10 / \beta_{0}$, and then released to adopt its final equilibrium shape. (b) Recovery ratio as a function of time for cases with initial stretch ratio of $\lambda_{0}=1.1,1.2$ and 1.5. Inset shows corresponding stress relaxation. Parameters used here are: $c=0.30$ (filaments with $L_{c} / l_{p}=5 / 3$ ), $x=0.65$ (a small pre-tensile stress, since $x_{0}=0.63$ of force-free state) and contour length of subfilament is $L_{c}=10^{3} a\left(l_{p}=600 a\right)$.

after release, the cell can recover its natural shape of biconcave plate if $t$ is short $(\sim 1 \mathrm{~min})$, while keeping the shape of the slit instead of recovering if $t$ is long ( $\sim 5 \mathrm{~min})$. Shape recovery of the cell is due to self-organisation of its cytoskeleton: when being deformed, crosslinks of the cytoskeleton are dynamically broken and re-formed, i.e., a new network structure under external deformation is formed if the time is long enough compared with the time scale for crosslink breakage.

Let us assume that at time $t=0$, the network is uniaxially stretched in $x$ direction instantaneously to a elongation ratio, $\lambda_{0}$, while the $y$ and $z$ directions experience identical compression with the ratio, $1 / \sqrt{\lambda_{0}}$, as shown in Fig. 2 (a). In this case, the breakage rate of the crosslinks becomes $\beta\left(0^{+} ; 0\right)$, where time $t=0^{+}$denotes for the state after stretching with elongation ratio $\lambda_{0}$, and time $t=0$ denotes for the relaxed state before stretching. This rate is a function of $\lambda_{0}$, and remains constant in a uniaxial stretch test with constant $\lambda_{0}$. Accordingly, the stress will decay exponentially with time. The reason is that this relaxation is essentially the plastic flow (creep) due to the breakage of original crosslinks, so only the 
first term in Eq. (4) contributes to the elastic stress, which directly leads to the relation

$$
\boldsymbol{\sigma}_{\text {ela }}(t)=\sigma\left(\lambda_{0}\right) e^{-\beta\left(0^{+} ; 0\right) t}
$$

where $\sigma\left(\lambda_{0}\right)=\partial F_{\text {p.n }} /\left.\partial \lambda\right|_{\lambda_{0}}$ at constant applied $\lambda_{0}$, with $F_{\text {p.n }}$ defined in Eq. (2). There is no contribution from the chains that were re-crosslinked after $t=0$ in Eq. (5), since their deformation state at any time $t$ with the externally imposed stretch ratio $\lambda_{0}$ is exactly the same as their reference state. This prediction of a simple-exponential stress decay is essentially the same as that of transient flexible network, 20 which is a natural outcome of the Tanaka-Edwards the crosslink dynamics. 16

If we release the initial load at time $t$, then the material will try to recover its equilibrium shape. The mechanical recovery is usually a fast process compared with the stress relaxation of plastic creep as above, so in the recovery process the material can be regarded as a permanent network without considering the furhter breakage and the re-connection of crosslinks. Let us define the equilibrium state after recovery to have the residual elongation ratio $\lambda_{r}(t)$; its value can be obtained by equalising the residue stress of the transient network after relaxation [the right-hand side of Eq. (6)] and the stress of a permanent network with its reference state defined with the elongation ratio $\lambda_{r}(t)$ [the left-hand side of Eq. (6)]:

$$
\sigma\left[\lambda_{0} / \lambda_{r}(t)\right]=\sigma\left(\lambda_{0}\right) e^{-\beta\left(0^{+} ; 0\right) t}
$$

A material parameter called the 'recovery ratio' can be introduced to measure how much a material can recover towards its original reference state $(\lambda=1)$, after remaining stretched for a period of time $t$ :

$$
S(t)=\frac{\lambda_{0}-\lambda_{r}(t)}{\lambda_{0}-1}
$$

Here $S=1$ represents full recovery of a purely elastic body, and $S=0$ represents zero 
recovery, where the plastic flow has relaxed the tension to zero. As shown in Fig. 2(b), the recovery ratio decreases with time, as more original crosslinks are broken with time, corresponding to stress relaxation as shown in the inset; material loses memory by a plastic flow. A characteristic time scale is used for the plot: $1 / \beta_{0} \sim 1 \mathrm{~min}$, which is the average time for a chain to spontaneously break from its crosslink.

\section{Neck formation}

Let us examine how the material responds under a ramp of uniaxial extension, when the principal stretching ratio increases with time: $\lambda=1+\dot{\gamma}$, with a constant rate $\dot{\gamma}$. We only find a small quantitative change in the curves of stress-strain relations when the rate of re-crosslinking changes: all the same qualitative features are maintained across the range of $\beta / \rho=10.0$ to $\beta / \rho=0.1$. This lack of dependence on re-crosslinking rate appears to be a common feature of the networks with finite stretchability of their strands. The main reason is that the elastic contribution of the newly crosslinked chains, which are by construction not far from their reference state, turns out negligible compared with the contribution from chains crosslinked from the beginning, especially when the initially crosslinked chains are being stretched up to the limit $x \rightarrow 1.21$ In the following discussion, we will take the limit $\beta \gg \rho$ for simplicity.

If the chains cannot be re-crosslinked for other reasons (e.g. without ATP supply to crosslinkers of an actin network ${ }^{(5)}$ ), the stress-strain relationship of a breakable network can be simplified by only using the first term in Eq. (4):

$$
\sigma_{x}(\lambda(t) ; t)=\frac{\partial F_{\mathrm{p} . \mathrm{n}}(\lambda(t) ; t)}{\partial \lambda} \exp \left[-\int_{0}^{t} \beta\left(t^{\prime} ; 0\right) d t^{\prime}\right]
$$

while $\sigma_{y}=\sigma_{z}=0$ remains for free boundaries. There are two main differences between Eq. (5) and Eq. (8): the immediate stress, $\partial F_{\mathrm{p} . \mathrm{n}} / \partial \lambda$, is increasing with time during the ramp deformation, as in Eq. (8) with $\lambda=1+\dot{\gamma}$, while it is constant in Eq. (5) during stress 

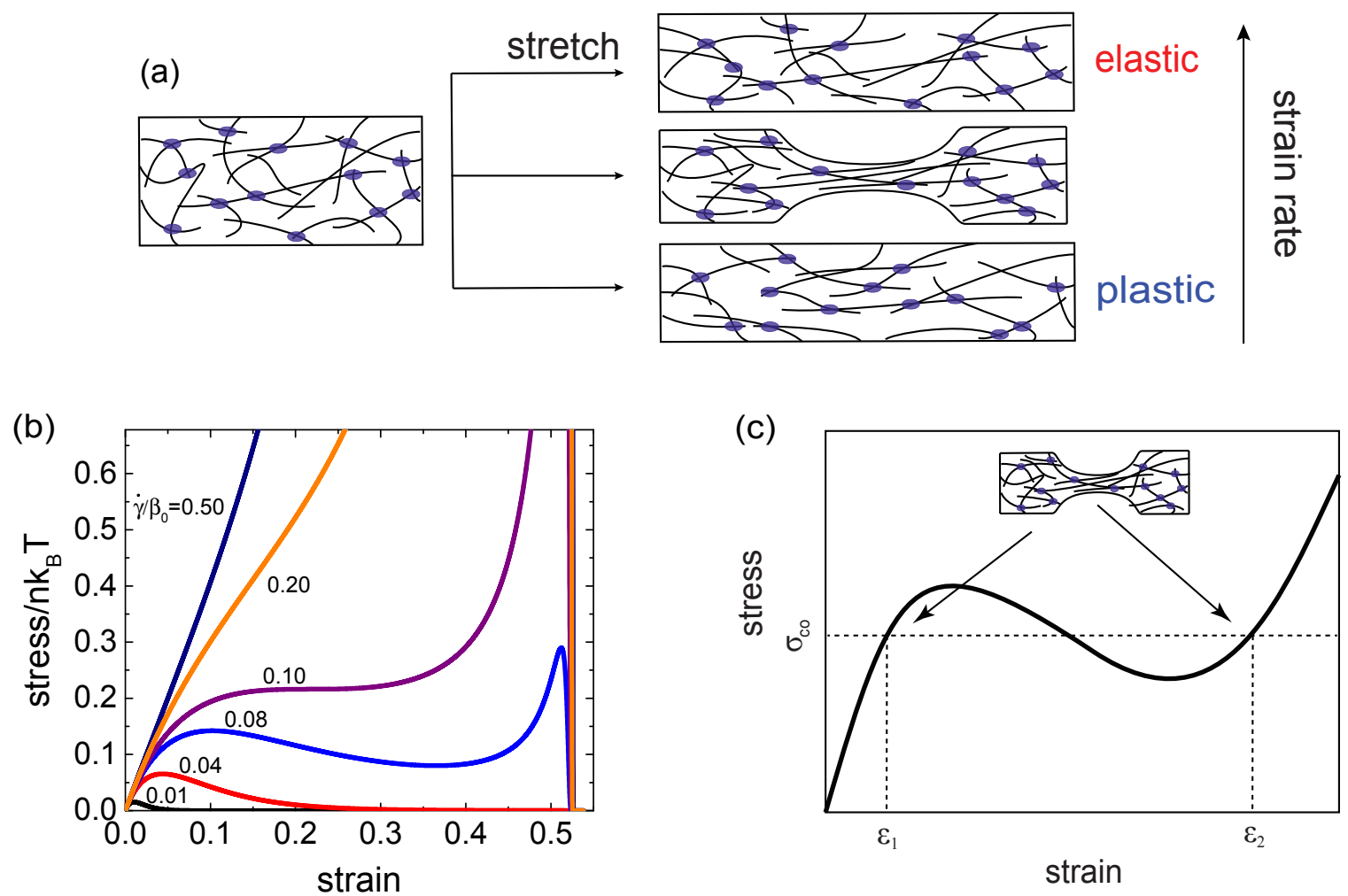

Figure 3: (a) Sketches of a transient filament network under uniaxial stretch, deforming elastically, showing a necking instability, or undergoing a plastic flow (creep). (b) Stressstrain relationship, with two critical strain rates: $\dot{\gamma}_{c 1}=0.04 \beta_{0}$ and $\dot{\gamma}_{c 2}=0.10 \beta_{0}$ (see text for detail). (c) A sketch of necking instability. Parameters used here are: $c=0.30$ (filaments with $L_{c} / l_{p}=5 / 3$ ), $x=0.65$ (a small pre-tensile stress, since $x_{0}=0.63$ of force-free state) and contour length of subfilament is $L_{c}=10^{3} a\left(l_{p}=600 a\right)$.

relaxation with fixed $\lambda_{0}$. Also, the rate of crosslink breaking $\beta(t ; 0)$ is changing with time in a ramp deformation, while it remains a constant in stress relaxation.

Tensile stress of a permanent filament network increases monotonously with strain. In contrast, the stress in a transient filament network under a deformation ramp can reach a yield point and subsequently decrease, as shown in Fig. 3(b). This is due to the onset of plastic flow, which was also spotted in the extended glassy worm-like chain model ${ }^{24}$. The reduced strain rate $\dot{\gamma} / \beta_{0}$ compares the time scale of the imposed deformation with that of internal crosslink dynamics. There are three response regimes depending on $\dot{\gamma} / \beta_{0}$, and two 
critical strain rates separating these three regimes defined as $\dot{\gamma}_{c 1}$ and $\dot{\gamma}_{c 2}$. In the case shown in Fig. 3(b) with the filament parameters (stiffness) $c=0.30$ and (pre-tension) $x=0.65$, the two critical strain rate are $\dot{\gamma}_{c 1}=0.04 \beta_{0}$ and $\dot{\gamma}_{c 2}=0.10 \beta_{0}$. If the strain rate is very low, $\dot{\gamma}<\dot{\gamma}_{c 1}$, the stress initially increases, but then decreases with the growing strain when the network undergoes a complete fluidization. The characteristic "stress overshoot" (the point of yield stress, in other terminology) also depends on the ratio $\dot{\gamma} / \beta_{0}$. For a relatively large strain rate, $\dot{\gamma}>\dot{\gamma}_{c 2}$, the stress increases nonlinearly but monotonically with the growing strain, until the whole material approaches its stretch limit (some chains become stretched with $x \rightarrow 1$ ), where the stress firstly increases in a almost diverging way and then abruptly falls to zero due to the breakage of all crosslinks under infinitely large forces, see Eq. (1).

Under a moderate strain rate (of a constant strain ramp considered here), in the range between $\dot{\gamma}_{c 1}$ and $\dot{\gamma}_{c 2}$, the stress first increases towards the yield point, then decreases, and then follows another increase - until the final breakdown of the network. The mechanism for the first increase and decrease in the stress is same as for the low strain rate case. The second increase in the stress is due to elasticity divergence for $x \rightarrow 1$, which is contributed by the chains remaining crosslinked from the beginning, similarly with that diverging increase under large strain rate as discussed above. When this occurs as a result of the competition between plasticity and nonlinear hyper-elasticity at $x \rightarrow 1$, there will be two coexisting phases with different strains (a weakly stretched one $\varepsilon_{1}$ and a strongly stretched one $\varepsilon_{2}$ ), and an identical stress throughout the material, $\sigma_{\mathrm{co}}$, shown in Fig. 3(c). The coexisting strains and stress can be obtained by the Maxwell construction for typical first-order phase transition, e.g., gas-liquid transition. In other words, a neck-like region will form in the material, where the highly-stretched part of the network has the same tensile stress as the matching weakly-stretched part. Actually, necking can also be found for transient flexible polymer networks with finite stretchability, 21 while the only material parameter in that case is the stretchability limit of chains. This was seen experimentally by Wang et al. $\frac{14]}{1 n}$ the context of filamentous connective tissue: a highly stretched region and a region with 


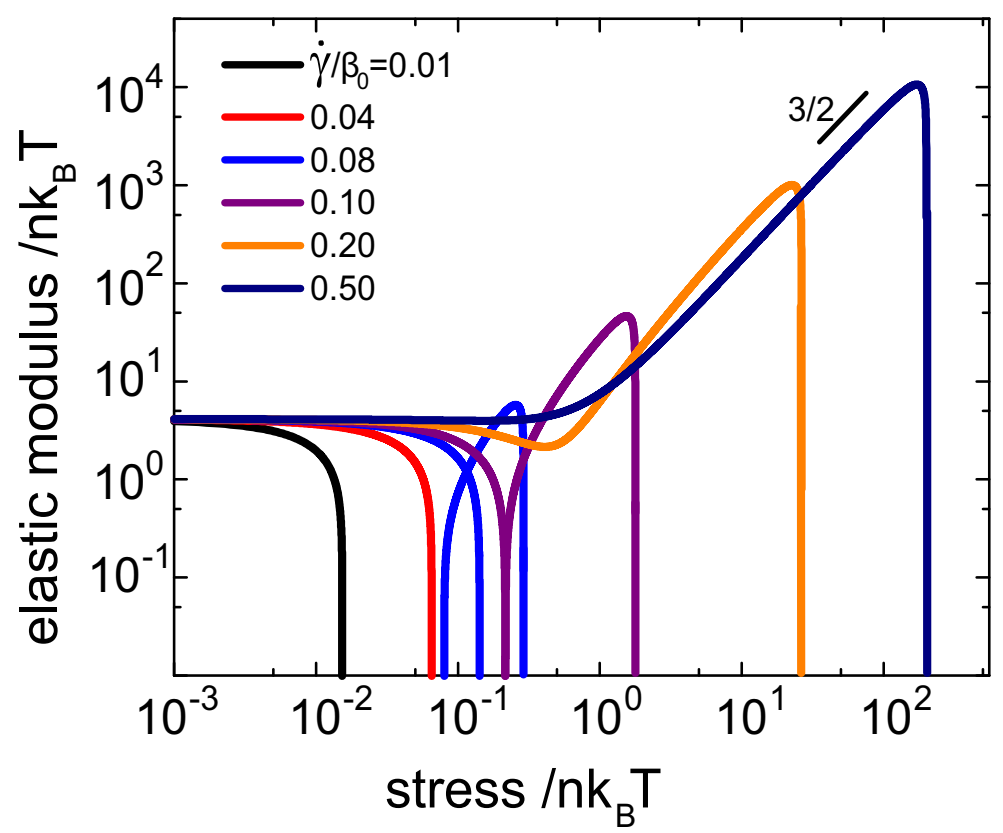

Figure 4: Elastic modulus-stress relationship of a transient network under uniaxial deformation ramp with different strain rates, measured against the natural rate of crosslink breaking $\beta_{0}$. Parameters used here are: $c=0.30$ (filaments with $L_{c} / l_{p}=5 / 3$ ), $x=0.65$ (a small pre-tensile stress, since $x_{0}=0.63$ of force-free state) and contour length of subfilament is $L_{c}=10^{3} a\left(l_{p}=600 a\right)$.

low deformation will coexist at the same local stress. In fact, the necking instability is a phenomenon broadly known across many fields of material science. $\underline{44} 47$

For a permanently crosslinked filament network, $27 \mid 48$ the $\operatorname{differential~modulus~} G$ is a constant when the stress $\sigma$ in the network is small, but undergoes a characteristic increase $G \sim \sigma^{3 / 2}$ for large deformation (stress-stiffening). This effect is determined by the mechanical properties of an individual filament, as its free energy is inversely proportional to $\left(1-x^{2}\right)$ for large deformation, when $x \rightarrow 1 . \sqrt[29|48| 53]{ }$ For a transient network, it is found that when the deformation rate is high ( say $\dot{\gamma}=0.50 \beta_{0}$ ), the network responds in the same way as the permanent network: its linear elastic modulus is almost a constant for small stress, and then the modulus grows with $\sim \sigma^{3 / 2}$ scaling for large stress, before the final failure of the material, see Fig 4. If the deformation rate is lower, but the mechanical response is still elastic (say $\dot{\gamma}=0.20 \beta_{0}$ in Fig 4 ), the shear modulus can initially decrease the growing stress (showing a dynamic stress-softening), but then return to a growing function $G \sim \sigma^{3 / 2}$ for 
larger stress. This late increase is due to the rapid growth of the elastic free energy as $x \rightarrow 1$, which overtakes the initial crosslink "slip".

In the regime where there is a neck-like region forming, between the two $\dot{\gamma}_{c 1}$ and $\dot{\gamma}_{c 2}$ curves in Fig. 3(b), the response in $G(\sigma)$ is qualitatively different. Initially the linear modulus decreases in the same way, in the stress-softening process we discussed earlier, however, $G$ becomes zero (at the yield point) and then negative in the region of instability, before starting to grow again, see Fig. 3(b) for a stress-strain representation. In the modulus-stress curves of Fig. 4 this manifests itself as a two-valued plot when the two branches of the network (the necking highly-stretched, and the weakly-stretched) coexist at the same stress. Finally, at a large stress the modulus enters into the familiar $3 / 2$ scaling stiffening regime, unless the network breakdown occurs earlier. At a very low deformation rate $\left(\dot{\gamma}<\dot{\gamma}_{c 1}\right)$, the elastic modulus decreases all the way to zero with the increase of the stress, without showing the $3 / 2$ scaling law any more. That is, the network loses its stability and the material fluidises at a moderate stress.

A full phase diagram in the space of relative stiffness (measuired by $c$ ), pre-tension of crosslinked filaments (measured by $x \neq x_{0}$ ), and the applied strain rate $\dot{\gamma}$ is shown in Fig. 5, where the orange cylinder-like zone marks the mechanically unstable region, where the filament network becomes fluid with increasing stiffness and decreasing pre-tension). The blue surface separates those of plasticity and necking (corresponding to $\dot{\gamma}_{c 1}$ branch) in Fig. 3), and the red surface separates the regimes of elasticity and necking (corresponding to $\dot{\gamma}_{c 2}$ branch. Plasticity and necking instability region increases with increasing pre-tension $x$ and decreasing stiffness $c$. Note the dashed line on the $c-x$ plane, marking the force-free state of the filament network $x_{0}(c)$.

\section{Polydisperse network}

Practically, the mesh size in a network is not uniform, but varies randomly with a certain distribution. We now discuss the influence of this polydispersity in the mesh size on the 


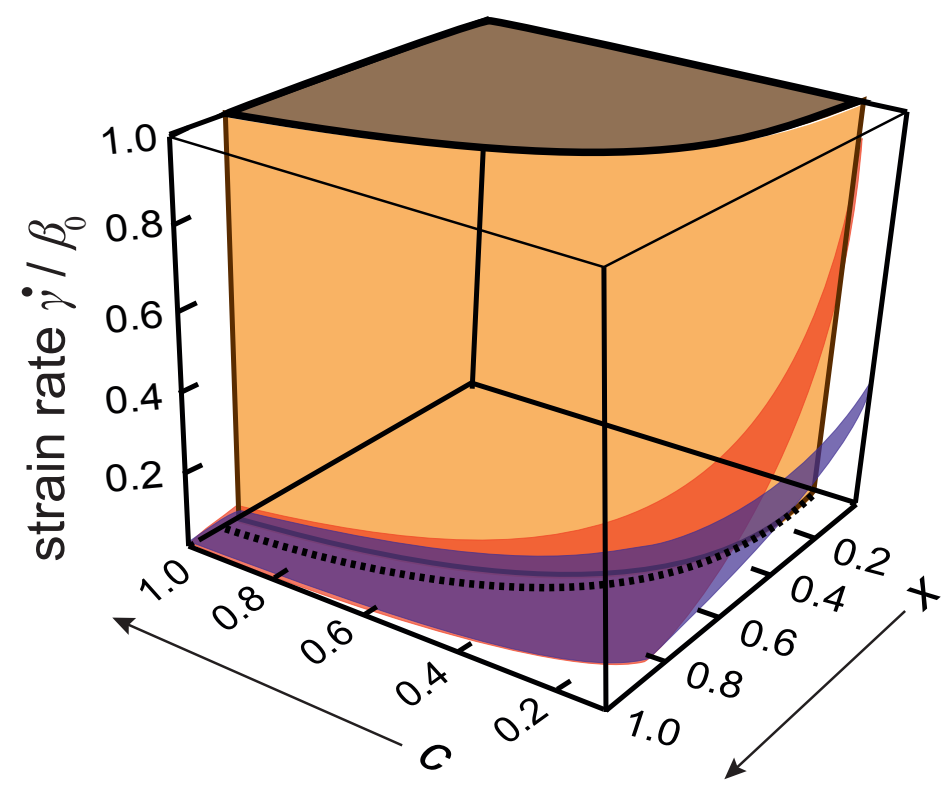

Figure 5: 3D phase diagram in the space of (stiffness, pre-stress, strain rate) of how a transient network responds to uniaxial stretch. Red surface denotes the boundary between necking and elasticity regions, blue surface denotes the boundary between necking and plasticity regions, orange cylinder-like zone denotes the unstable region (below the marginal rigidity threshold), and the dashed line in the $c-x$ plane denotes for force-free condition $x_{0}(c)$ of the filaments. Contour length of subfilament is $L_{c}=10^{3} a$.

rheological properties of transient filament networks in this section.

The probability to find a sub-filament connecting two crosslinks with length $L_{c}$ under the assumption of normal distribution is $p\left(L_{c}\right)=(1 / \sqrt{2 \pi} \Delta) \exp \left[-\left(L_{c}-L_{c}^{0}\right) / 2 \Delta^{2}\right]$, with the expectation value $L_{c}^{0}$ and standard variance $\Delta$, where the variance can be controlled in experiments. 11 The mean length $L_{c}^{0}$ is related to the number density of crosslinks $n$ via $L_{c}^{0} \sim n^{-1 / 3}$. Free energy of transient filament network can be obtained by adding the contributions from all the sub-networks of different mesh size:

$$
F_{\mathrm{t} . \mathrm{n}}(t)=\int F_{\mathrm{t} . \mathrm{n}}\left(t ; L_{c}\right) p\left(L_{c}\right) d L_{c} .
$$

For simplicity of numerical analysis, we will assume chains in cells experiencing large enough stress with $\sigma\left(L_{c}\right) / n k_{\mathrm{B}} T \geq 1000$ will break from crosslinks, rather than keeping an infinitesi- 
mal probability of them remaining not broken. Moreover, we will only discuss the properties of a transient network with its initial crosslinked state force-free as an example; in other words, a sub-network with different sub-filament contour length has a corresponding mesh size, $\xi\left(L_{c}\right)$, for achieving the force-free state of each single filament (with different lengths).

\section{Non-exponential stress relaxation}

A non-exponential stress relaxation is found in a transient network with polydisperse subfilament length / mesh size. Figure 6(a) illustrates the time dependence of stress relaxation under a constant strain $\lambda_{0}=1.1$, comparing the monodisperse network $(\Delta=0)$ with the cases of increasing polydispersity. In polydisperse network the elastic stress decays faster at the beginning [inset of Fig. 6(a)], and becomes slower in a transient network with a broader distribution of mesh size. Due to the highly non-linear filament elasticity, the force acting on the filament from different-sized meshes is different upon the same deformation, and it decreases with the increase of the mesh size, as being further to the divergence region of the free energy. Filaments experiencing larger forces would break off their crosslinks earlier, resulting in a faster decrease in the stress at the beginning of relaxation. With the ongoing time, the number of crosslinked filaments with a broader distribution then becomes smaller, which causes a slower decay of the stress, and eventually all curves end up with the simple exponential relaxation of the remaining nearly-monodisperse chains (although this regime may not be accessible in practice).

A stretched exponential decay in a transient network, expressed as $\exp \left[-(\beta t)^{\alpha}\right]$, is often reported in experiment, $\stackrel{54 / 55}{5}$ wich does not agree with the basic prediction of Eq. (5), but

could be explained by assuming polydispersity in the bond strength. $\frac{2056}{2}$ Here we assume polydispersity in the contour length of subchains (mesh size) of the network, when the network is stretched beyond the lower limit, (defined by smallest $L_{c}$ ), i.e., $1 / x\left(L_{c}\right)>\lambda_{0}$, the stress decays fast at the beginning due to fast breakage of the short over-stretched chains from the crosslinks with $1 / x\left(L_{c}\right) \rightarrow \lambda_{0}$, and $\alpha$ is small at short time scales and becomes 

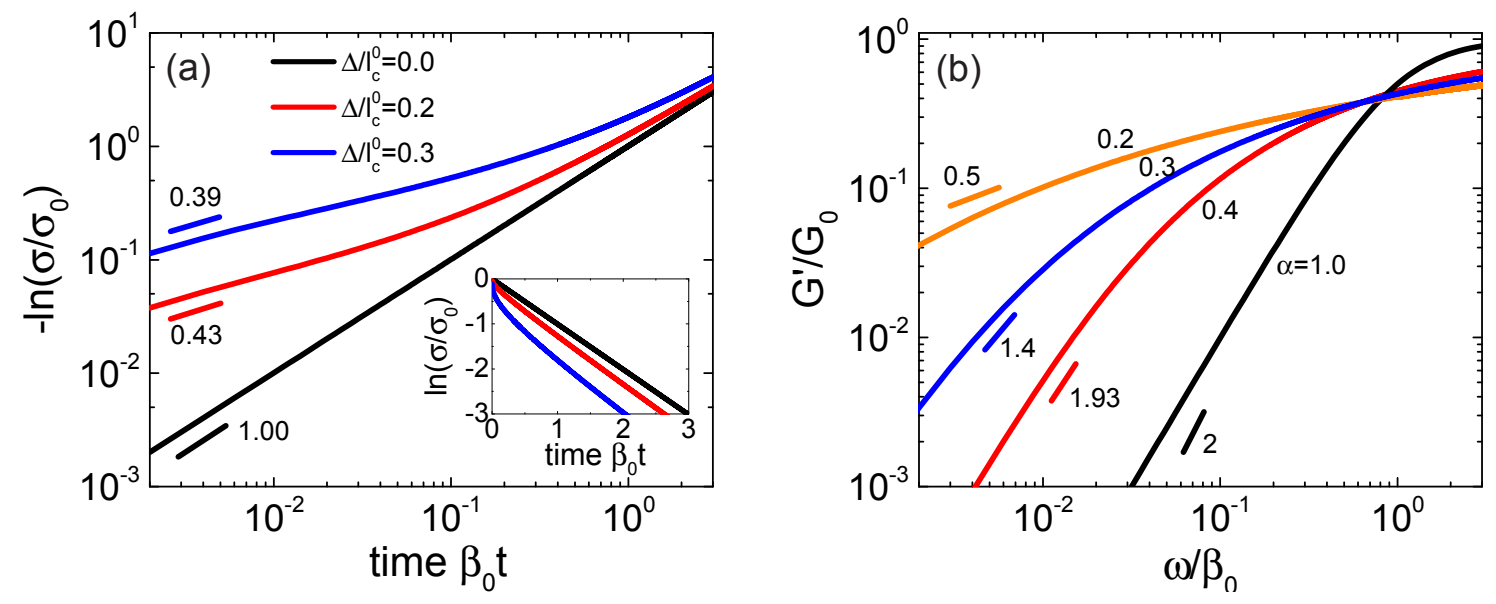

Figure 6: (a) Stress relaxation of transient networks with different distribution variances, $\left[\beta t,-\ln \left(\sigma / \sigma_{0}\right)\right]$ in log-log scale with the slope as the stretching factor $\alpha$ labelled on the curves. The inset displays the $\left[\beta t, \ln \left(\sigma / \sigma_{0}\right)\right]$ relation in log-linear scale. Parameters used are: pre-strain of $\lambda_{0}=1.1$, zero pre-tension with $x\left(L_{c}\right)=x_{0}\left(L_{c}\right)$, average contour length $L_{c}^{0}=1.25 l_{p}\left(c_{0}=0.40\right)$ and persistence length $l_{p}=800 a$. (b) Frequency dependence of $G^{\prime}$ for networks with various stretched factor $\alpha$ in stress relaxation, $\sigma \sim \exp \left[-(\beta t)^{\alpha}\right]$.

larger at longer ones, Fig. 6(a).

If we assume the modulus (or the stress) of the network decays as a stretched exponential, i.e., $G=G_{0} \exp \left[-(\beta t)^{\alpha}\right]$, then the storage and the dissipative modulus can be calculated by $G^{\prime}(\omega)=G_{0} \int_{0}^{\infty} \exp \left[-(\beta t)^{\alpha}\right] \omega \sin \omega t \mathrm{~d} t$, and $G^{\prime \prime}(\omega)=G_{0} \int_{0}^{\infty} \exp \left[-(\beta t)^{\alpha}\right] \omega \cos \omega t \mathrm{~d} t$, respectively. Given the stretching factor, $\alpha$, the frequency dependence of $G^{\prime}, G^{\prime \prime}$ can be calculated numerically, as shown in Fig. 6(b). We find that the larger is the stretching factor $\alpha$, the smaller is the exponent $\nu$ in $G^{\prime} \sim \omega^{\nu}$ in the low-frequency regime. Experimentally reported $\nu=0.5$ for long-time mechanic responses ${ }^{6 / 57}$ is recovered in the case of $\alpha=0.2$, which coincidentally is the stretching factor in some studies of the stress relaxation in transient flexible networks. $\frac{54}{54}$ This could be the actual mechanism for achieving $\nu=0.5$ in experiments.

\section{Stress-strain under ramp deformation}

As shown in Fig. 3(b), if strain rate is high, there is an instant fall of the elastic stress from a finite value to zero, as material breaks down when the deformation is approaching the 

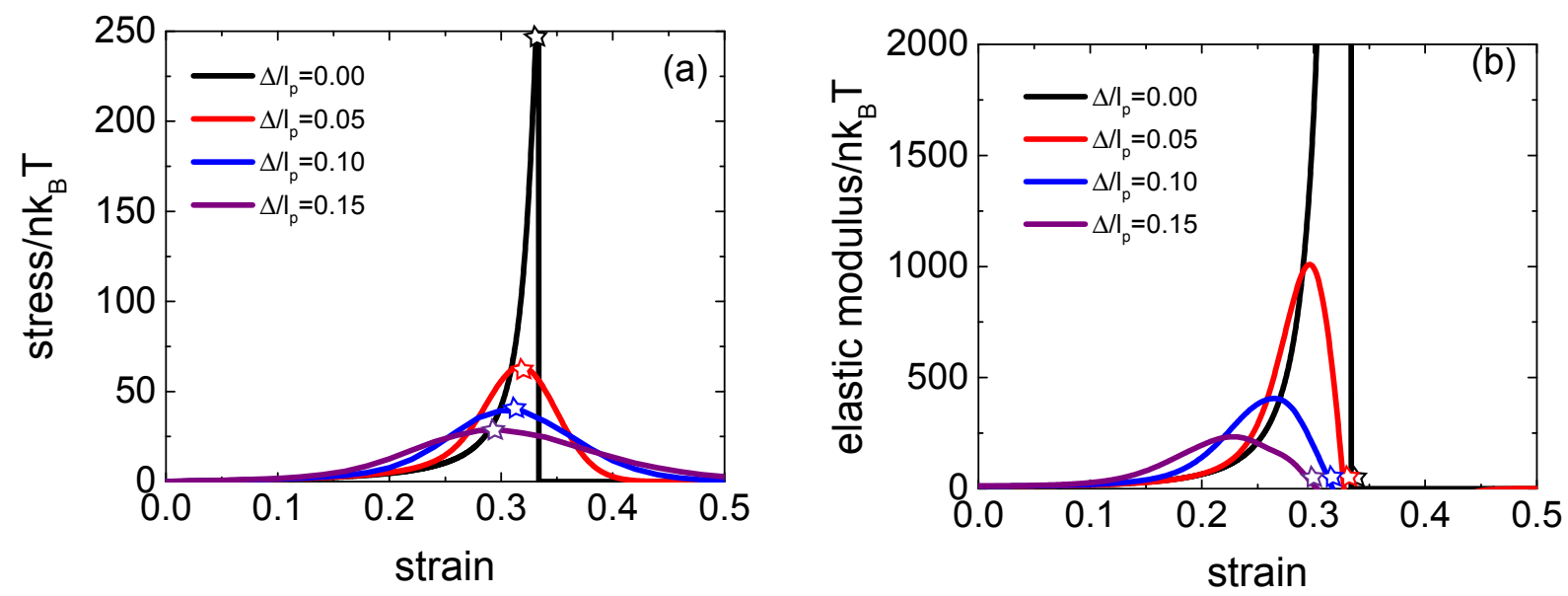

Figure 7: (a) Stress-strain relationship of a transient network with different distribution variances. (b) Modulus-strain relationship of a transient network with different distribution variances, both in the case of ramp stretch. Stars mark yield points, or elastic-plastic transition points. Parameters used are: strain rate $\dot{\gamma}=0.50 \beta_{0}$, zero pre-tension with $x\left(L_{c}\right)=x_{0}\left(L_{c}\right)$, average contour length, $L_{c}^{0}=1.25 l_{p}\left(c_{0}=0.40\right)$ and persistence length $l_{p}=800 a$.

filament limit, i.e. $x \rightarrow 1$. However, the breakdown process of the material observed in experiments is a bit different: $\frac{10[11}{1 t}$ it shows a smooth downturn, rather than a discontinuous fall in the stress. Similar smoothing is also found in the regime of plasticity and necking instability, but we will focus on the elasticity regime (high strain rate) as an example in the following discussion.

Polydispersity of the network can naturally explain the observations. As shown in Fig. 7 , downturns can become smooth in both the stress and the elastic modulus in polydisperse transient network, and the downturns happen at deceasing strains with increasing distribution variances; this is due to the earlier breakage of the sub-networks with smaller mesh sizes. Moreover, under a same strain, the stress firstly increases with the increased width of the distribution, if the strain is small, then decreases when the material is deformed up to its limit. That is, the material with a wide distribution in its mesh size hardens at small deformation, and softens at large deformation. The stress hardening at small deformation is because the sub-networks with smaller mesh size dominate in the contributions to the total stress, and these networks fluidize with time, with minor contributions at large deformation. 
As the small-mesh-sized sub-networks break, the one sized around $\xi_{0}$, with large distribution probabilities, determines how the material responds; the material with smaller variance in mesh size distribution has more sub-networks sized around $\xi_{0}$, so the stress of such material is larger, i.e., the material with smaller variance hardens at large deformation, especially when $x_{0} \rightarrow 1$.

\section{Conclusions}

In this work, we present a continuum theory for describing rheological properties of a transient filament network under arbitrary deformations, which contains dynamically broken and re-formed crosslinks. By defining reference states of a sub-network according to when it (or the corresponding crosslink) is formed, we can obtain the total free energy of the transient network as a sum of all sub-networks formed at different time. As an illustration, we show an exponential stress relaxation for the network containing uniform-sized mesh, and discuss how the material responds under a ramp deformation, where the network experiences a change from small to large deformations. In this type of experiment, three deformation regimes (elasticity, plasticity, and necking instability) are observed. We also extended the theory to a network with polydisperse mesh sizes, whose distribution is assumed to be Gaussian. In this case, a stretched exponential decay of the stress under a constant deformation strain is observed, changing as a function of the standard variance of the distribution; meanwhile, the frequency dependence of the storage (loss) modulus is discussed. Moreover, smooth yielding, stress hardening or softening, which is influenced by the distribution of the mesh sizes, is discussed in this work.

Here we applied affine deformation to all sub-units as an approximation, which is not

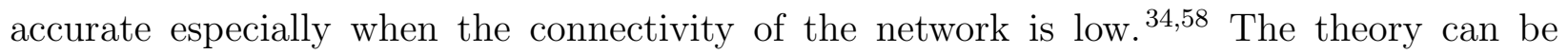
generalized to deal with cases of non-affine deformation, considering the effects of the local force relaxation around crosslinks. We believe that this model provides a basic understanding 
of a transient filament network containing breakable crosslinks, which is very portable and can be easily adapted to meet practical demands, and hope to stimulate more quantitative experiments on exploring the rheology of transient filament networks.

\section{Acknowledgement}

The authors thank Masao Doi, Alessio Zaccone, and ZhongCan OuYang for fruitful discus-

sions. This work is funded by the Theory of Condensed Matter Critical Mass Grant from EPSRC (EP/J017639).

\section{References}

(1) Pritchard, R. H.; Huang, Y. Y. S.; Terentjev, E. M. Mechanics of biological networks: From the cell cytoskeleton to connective tissue. Soft Matter 2014, 10, 1864-1884.

(2) Broedersz, C. P.; MacKintosh, F. C. Modeling semiflexible polymer networks. Rev. Mod. Phys. 2014, 86, 995-1036.

(3) Meng, F.; Terentjev, E. Theory of semiflexible filaments and networks. Polymers 2017, 9,52 .

(4) Wachsstock, D.; Schwarz, W.; Pollard, T. Cross-linker dynamics determine the mechanical properties of actin gels. Biophys. J. 1994, 66, 801-809.

(5) Lieleg, O.; Claessens, M. M. A. E.; Luan, Y.; Bausch, A. R. Transient binding and dissipation in cross-linked actin networks. Phys. Rev. Lett. 2008, 101, 1-4.

(6) Broedersz, C. P.; Depken, M.; Yao, N. Y.; Pollak, M. R.; Weitz, D. A.; MacKintosh, F. C. Cross-Link-Governed Dynamics of Biopolymer Networks. Phys. Rev. Lett. 2010, 105, 238101. 
(7) Humphrey, D.; Duggan, C.; Saha, D.; Smith, D.; Käs, J. Active fluidization of polymer networks through molecular motors. Nature 2002, 416, 413-416.

(8) Lieleg, O.; Kayser, J.; Brambilla, G.; Cipelletti, L.; Bausch, A. R. Slow dynamics and internal stress relaxation in bundled cytoskeletal networks. Nat. Mater. 2011, 10, 236242.

(9) Nam, S.; Hu, K. H.; Butte, M. J.; Chaudhuri, O. Strain-enhanced stress relaxation impacts nonlinear elasticity in collagen gels. Proc. Natl. Acad. Sci. 2016, 113, 201523906.

(10) Tharmann, R.; Claessens, M. M. A. E.; Bausch, A. R. Viscoelasticity of Isotropically Cross-Linked Actin Networks. Phys. Rev. Lett. 2007, 98, 088103.

(11) Jensen, M. H.; Morris, E. J.; Gallant, C. M.; Morgan, K. G.; Weitz, D. A.; Moore, J. R. Mechanism of calponin stabilization of cross-linked actin networks. Biophys. J. 2014, 106, 793-800.

(12) Yao, N. Y.; Becker, D. J.; Broedersz, C. P.; Depken, M.; MacKintosh, F. C.; Pollak, M. R.; Weitz, D. A. Nonlinear Viscoelasticity of Actin Transiently Cross-linked with Mutant $\alpha$-Actinin-4. J. Mol. Biol. 2011, 411, 1062-1071.

(13) Murakami, R.; Tsai, C. H. D.; Ito, H.; Tanaka, M.; Sakuma, S.; Arai, F.; Kaneko, M. Catch, load and launch toward on-chip active cell evaluation. 2016 IEEE International Conference on Robotics and Automation (ICRA). 2016; pp 1713-1718.

(14) Wang, H.; Svoronos, A. a.; Boudou, T.; Sakar, M. S.; Schell, J. Y.; Morgan, J. R.; Chen, C. S.; Shenoy, V. B. Necking and failure of constrained 3D microtissues induced by cellular tension. Proc. Natl. Acad. Sci. USA 2013, 110, 20923-8.

(15) Green, M. S.; Tobolsky, A. V. A new approach to the theory of relaxing polymeric media. J. Chem. Phys. 1946, 14, 80-92. 
(16) Tanaka, F.; Edwards, S. Viscoelastic Properties of Physically Cross-Linked Networks. Transient Network Theory. 1992, 25, 1516-1523.

(17) Drozdov, A. D. A constitutive model in finite thermoviscoelasticity based on the concept of transient networks. Acta Mechanica 1999, 133, 13-37.

(18) Hui, C.-Y.; Long, R. A constitutive model for the large deformation of a self-healing gel. Soft Matter 2012, 133, 8209-8216.

(19) Long, R.; Mayumi, K.; Creton, C.; Narita, T.; Hui, C.-Y. Rheology of a dual crosslink self-healing gel. J. Rheol. 2015, 59, 643-665.

(20) Meng, F.; Pritchard, R. H.; Terentjev, E. M. Stress relaxation, dynamics, and plasticity of transient polymer networks. Macromolecules 2016, 49, 2843-2852.

(21) Meng, F.; Terentjev, E. Transient network at large deformations: elastic-plastic transition and necking instability. Polymers 2016, 8, 108.

(22) Unterberger, M. J.; Schmoller, K. M.; Wurm, C.; Bausch, A. R.; Holzapfel, G. A. Viscoelasticity of cross-linked actin networks: Experimental tests, mechanical modeling and finite-element analysis. Acta Biomater. 2013, 9, 7343-7353.

(23) Wolff, L.; Fernández, P.; Kroy, K. Resolving the Stiffening-Softening Paradox in Cell Mechanics. PLOS ONE 2012, 7, e40063.

(24) Wolff, L.; Fernandez, P.; Kroy, K. Inelastic mechanics of sticky biopolymer networks. New J. Phys. 2010, 12, 053024.

(25) López-Menéndez, H.; Rodríguez, J. F. Microstructural model for cyclic hardening in F-actin networks crosslinked by $\alpha$-actinin. J. Mech. Phys. Sol. 2016, 91, 28-39.

(26) Palmer, J. S.; Boyce, M. C. Constitutive modeling of the stress-strain behavior of Factin filament networks. Acta Biomaterialia 2008, 4, 597-612. 
(27) Meng, F.; Terentjev, E. M. Nonlinear elasticity of semiflexible filament networks. Soft Matter 2016, 12, 6749-6756.

(28) Kratky, O.; Porod, G. Röntgenuntersuchung gelöster Fadenmoleküle. Recueil des Travaux Chimiques des Pays-Bas 1949, 68, 1106-1122.

(29) Blundell, J. R.; Terentjev, E. M. Stretching semiflexible filaments and their networks. Macromolecules 2009, 42, 5388-5394.

(30) Boyce, M. C.; Arruda, E. M. Constitutive models of rubber elasticity: a review. Rubber Chem. Technol. 2000, 73, 504-523.

(31) Treloar, L. R. G. The Physics of Rubber Elasticity; Oxford University Press: Oxford, 1975.

(32) Janmey, P. A.; McCormick, M. E.; Rammensee, S.; Leight, J. L.; Georges, P. C.; MacKintosh, F. C. Negative normal stress in semiflexible biopolymer gels. Nat. Mater. 2007, 6, 48-51.

(33) Kang, H.; Wen, Q.; Janmey, P. A.; Tang, J. X.; Conti, E.; MacKintosh, F. C. Nonlinear elasticity of stiff filament networks: Strain stiffening, negative normal stress, and filament alignment in fibrin gels. J. Phys. Chem. B 2009, 113, 3799-3805.

(34) Sharma, A.; Licup, A. J.; Jansen, K. A.; Rens, R.; Sheinman, M.; Koenderink, G. H.; MacKintosh, F. C. Strain-controlled criticality governs the nonlinear mechanics of fibre networks. Nat. Phys. 2016, 12, 584-587.

(35) Bower, A. F. Applied mechanics of solids; CRC press: Boca Raton, 2009.

(36) Bell, G. I. Models for the specific adhesion of cells to cells. Science 1978, 200, 618-627.

(37) Guo, B.; Guilford, W. H. Mechanics of actomyosin bonds in different nucleotide states are tuned to muscle contraction. Proc. Natl. Acad. Sci. USA 2006, 103, 9844-9849. 
(38) Marshall, B. T.; Long, M.; Piper, J. W.; Yago, T.; McEver, R. P.; Zhu, C. Direct observation of catch bonds involving cell-adhesion molecules. Nature 2003, 423, 190-3.

(39) Tanaka, F.; Edwards, S. Viscoelastic properties of physically crosslinked networks Part 1. Non-linear stationary viscoelasticity. J. Non-Newtonian Fluid Mech. 1992, 43, 247271.

(40) Vandoolaeghe, W. L.; Terentjev, E. M. A Rouse-tube model of dynamic rubber viscoelasticity. J. Phys. A: Math. Theor. 2007, 40, 14725-14744.

(41) Farge, E.; Maggs, A. C. Dynamic scattering from semiflexible polymers. Macromolecules 1993, 26, 5041-5044.

(42) Kroy, K. Dynamics of wormlike and glassy wormlike chains. Soft Matter 2008, 4, 23232330.

(43) Brighenti, R.; Vernerey, F. J. A simple statistical approach to model the time-dependent response of polymers with reversible cross-links. Composites Part B: Engineering 2017, $115,257-265$.

(44) Eastgate, L. O.; Langer, J. S.; Pechenik, L. Dynamics of large-scale plastic deformation and the necking instability in amorphous solids. Phys. Rev. Lett. 2003, 90, 045506.

(45) Needleman, A. Continuum mechanics studies of plastic instabilities. Rev. Phys. Appl. 1988, 23, 585-593.

(46) Fielding, S. M. Criterion for extensional necking instability in polymeric fluids. Phys. Rev. Lett. 2011, 107, 258301.

(47) Kinloch, A.; Young, R. Fracture Behaviour of Polymers; Chapman \& Hall: London, 1995.

(48) Hinner, B.; Tempel, M.; Sackmann, E.; Kroy, K.; Frey, E. Entanglement, elasticity, and viscous relaxation of actin solutions. Phys. Rev. Lett. 1998, 12, 2614-2617. 
(49) Gardel, M. L.; Shin, J. H.; MacKintosh, F. C.; Mahadevan, L.; Matsudaira, P.; Weitz, D. A. Elastic behavior of cross-linked and bundled actin networks. Science 2004, 304, 1301-1305.

(50) Kouwer, P. H. J.; Koepf, M.; Le Sage, V. a. a.; Jaspers, M.; van Buul, A. M.; EksteenAkeroyd, Z. H.; Woltinge, T.; Schwartz, E.; Kitto, H. J.; Hoogenboom, R.; Picken, S. J.; Nolte, R. J. M.; Mendes, E.; Rowan, A. E. Responsive biomimetic networks from polyisocyanopeptide hydrogels. Nature 2013, 493, 651-655.

(51) Lin, Y. C.; Yao, N. Y.; Broedersz, C. P.; Herrmann, H.; MacKintosh, F. C.; Weitz, D. A. Origins of elasticity in intermediate filament networks. Phys. Rev. Lett. 2010, 104, 058101.

(52) Lin, Y. C.; Broedersz, C. P.; Rowat, A. C.; Wedig, T.; Herrmann, H.; MacKintosh, F. C.; Weitz, D. A. Divalent cations crosslink vimentin intermediate filament tail domains to regulate network mechanics. J. Mol. Biol. 2010, 399, 637-644.

(53) Gardel, M. L.; Shin, J. H.; MacKintosh, F. C.; Mahadevan, L.; Matsudaira, P. A.; Weitz, D. A. Scaling of F-actin network rheology to probe single filament elasticity and dynamics. Phys. Rev. Lett. 2004, 93, 188102.

(54) Hotta, A.; Clarke, S. M.; Terentjev, E. M. Stress relaxation in transient networks of symmetric triblock styrene-isoprene-styrene copolymer. Macromolecules 2002, 35, 271277.

(55) Séréro, Y.; Jacobsen, V.; Berret, J. F.; May, R. Evidence of nonlinear chain stretching in the rheology of transient networks. Macromolecules 2000, 33, 1841-1847.

(56) Drozdov, A. D. A model for the non-linear viscoelastic response and physical aging in glassy polymers. Int. J. Solids Struc. 1998, 35, 2315-2347. 
(57) Müller, K. W.; Bruinsma, R. F.; Lieleg, O.; Bausch, A. R.; Wall, W. A.; Levine, A. J. Rheology of Semiflexible Bundle Networks with Transient Linkers. Phys. Rev. Lett. 2014, 112, 238102.

(58) Ennomani, H.; Letort, G.; Guérin, C.; Martiel, J. L.; Cao, W.; Nédélec, F.; De La Cruz, E. M.; Théry, M.; Blanchoin, L. Architecture and Connectivity Govern Actin Network Contractility. Current Biol. 2016, 26, 616-626. 
Title: "Fluidization of Transient Filament Networks"

Author(s): Meng, Fanlong; Terentjev, Eugene

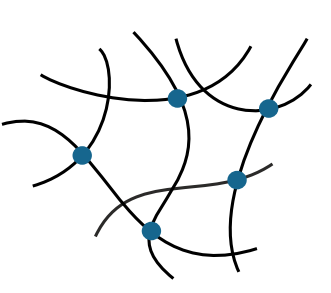

: breakable crosslinks
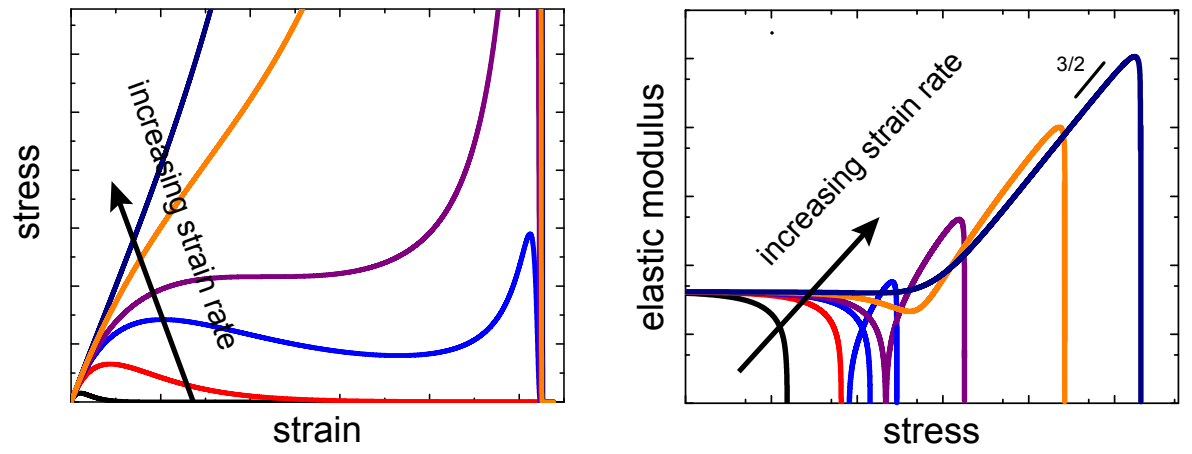

Figure 8: for Table of Contents use only. 